\title{
A nonstandard numerical scheme for a predator-prey model with Allee effect
}

\author{
Mevlude Yakit Ongun ${ }^{\mathrm{a}, *}$, Nihal Ozdogan ${ }^{\mathrm{b}}$ \\ a Suleyman Demirel University, Department of Mathematics, Isparta, Turkey. \\ b Suleyman Demirel University, Vocational School of Higher Education, Isparta, Turkey. \\ Communicated by A. Atangana
}

\begin{abstract}
In this paper, we present a Lotka-Volterra predator-prey model with Allee effect. This system with general functional response has an Allee effect on prey population. A nonstandard finite difference scheme is constructed to transform the continuous time predator-prey model with Allee effect into the discrete time model. We use the Schur-Cohn criteria which deal with coefficients of the characteristic polynomial for determining the stability of discrete time system. The proposed numerical schemes preserve the positivity of the solutions with positive initial conditions. The new discrete-time model shows dynamic consistency with continuous-time model. (C)2017 All rights reserved.
\end{abstract}

Keywords: Allee effect, stability analysis, nonstandard finite difference scheme, predator-prey model. 2010 MSC: 65L12, 65L20, 92B05.

\section{Introduction}

During the last few decades, the predator-prey dynamics have great interest in mathematical biology. Mathematical modelling is a useful tool for observe the dynamic behavior of these systems. According to this, a general predator-prey model is given by:

$$
\begin{aligned}
& \frac{d x}{d t}=p(x)-\operatorname{ayf}(x, y), \\
& \frac{d y}{d t}=y f(x, y)-\mu(y),
\end{aligned}
$$

where $x$ is the prey population sizes, $y$ is the predator population sizes, $p(x)$ is the intrinsic growth rate of the prey, $\mu(y)$ is the mortality rate of the predator, $a$ is the assimilation efficiency and $x(0) \geqslant 0, y(0) \geqslant 0$. The function $f(x, y)$ which found in some rare models is also called "functional response" and describes the number of prey consumed per predator unit time. Prey and predator equations of system (1.1) can appear in any order.

In the literature the predator mortality rate $\mu(y)$ depends linearly on the predator density $(\mu(y)=d y)$ while $p(x)$ has a linear $(p(x)=b x)$ or logistic $\left(p(x)=b x\left(1-\frac{x}{k}\right)\right)$ evolution. The most

\footnotetext{
*Corresponding author

Email addresses: mevludeyakit@sdu.edu.tr (Mevlude Yakit Ongun), nihalozdogan@sdu.edu.tr (Nihal Ozdogan)
} 
important thing is to state of the function $f(x, y)$ for us. The functional response functions have different types for example, Holling Type-I functional response [13, 14, 21], Holling Type-II functional response $[1,6,14,21]$, Holling Type-III functional response [14, 21, 27] are used in mathematical modelling. These are:

- Holling Type I: $f=\alpha x$;

- Holling Type II: $f=\frac{\alpha x}{x+c}$;

- Holling Type III: $f=\frac{\alpha x^{2}}{x^{2}+c}$.

Also better known as Lotka-Volterra prey-predator models are popular topics of the last year [19]. In predator-prey interactions where predators have to search for food are more appropriate on ratiodependent predator-prey models. The functional response function $f(x, y)$ as the ratio-dependent type $f=\frac{\alpha x}{x+b y}[4,5,17]$.

First time in 1931 the ecological importance of animal aggregations were revealed by the ecologist Allee. Thus, the effect which is called Allee effect, was described as a positive relation between individual convenience and population density. This effect may be caused by factors in, for example, predator defense or avoidance and food exploitation, inbreeding depression, social dysfunction, finding mates. So aggregated distributions of organisms over the available resources may show the presence of an Allee effect. In a predator-prey system, there may be two different types of Allee effects namely Allee effect I and Allee effect II. Allee effect I caused by increasing death rate or decreasing birth rate of the prey population, Allee effect II caused by precaution status or anti-predator defense.

It may cause difficulties in finding equilibrium points, searching local stability due to the effect which was found in both of them at the same time. Strong and weak Allee effect may be mentioned that as a result of the Allee effect has a huge impact for deterioration of biological balance and when this effect is neglected it can cause some problems. One of the most popular papers related to this effect can be read in $[7,19,25,28]$.

Numerical methods, such as Adams methods, Runge-Kutta and Euler methods based on finite difference approximations are frequently used to examine the dynamics of the interacting populations. But disadvantages of these methods are, their accuracy and stability depend on the time step-size. Because the discrete system obtained by the continuous system has truncation errors. Anguelov and Lubuma [2], Dimitrov and Kojouharov [8, 9] and Lubuma and Roux [18], among others, have used Nonstandard Finite Difference Schemes (NSFD), developed by Mickens [20] for designing methods that conserve the local stability of equilibria of the approximated system. However, the NSFD methods, guarantee positive discrete solution for positive initial conditions [11]. Recently, several stable and positive nonstandard finite difference methods have been improved for some specific predator-prey systems and epidemic systems $[3,9,10,16,22-24]$.

In this paper, we develop a new NSFD method for a general class of Lotka Volterra predator-prey system which type is Holling Type-II with Allee effect. The new NSFD method preserves the physical and biological properties, especially the stability of equilibria, of the approximated system.

The paper is organized as follows: In Section 2, we supply some basics and preliminary results. In Section 3, NSFD are developed for this system with general functional response and we present a stability analysis of steady-state solutions of a discrete time Holling Type-II Lotka Volterra predator-prey system with Allee effect. Furthermore, Jury conditions for local asymptotic stability analysis are tested. In Section 4 we illustrate our main results by numerical examples.

\section{Definitions and preliminaries}

Let

$$
\frac{\mathrm{d} z}{\mathrm{dt}}=\mathrm{F}(z), \quad z\left(\mathrm{t}_{0}\right)=z_{0} \geqslant 0 .
$$


In this equation, $z(t)=(x(t), y(t))^{\top}$ and the function $F=\left[F^{1}, F^{2}\right]^{\top}: \mathbb{R}^{2} \longrightarrow \mathbb{R}^{2}$ is differentiable. The equilibrium points of equation (2.1) are obtained as the solutions of $F(z)=0$. A general one-step numerical scheme with a step size $h$, that approximates the solution $z(t)$ of the system can be written in the form:

$$
\mathrm{D}_{\mathrm{h}}\left(z_{\mathrm{k}}\right)=\mathscr{F}_{\mathrm{h}}\left(\mathrm{F} ; z_{\mathrm{k}}\right),
$$

where $D_{h}\left(z_{k}\right) \approx\left(\frac{d x}{d t}, \frac{d y}{d t}\right)^{\top}$ and $\mathscr{F}_{h}\left(F ; z_{k}\right)$ approximates the right-hand side of system $(2.2), z_{k} \approx z\left(t_{k}\right)$ and $t_{k}=t_{0}+k h[9]$.

Definition 2.1. Let $z^{*}=\left(x^{*}, y^{*}\right)$ be an equilibrium of equation (2.1), J $\left(z^{*}\right)$ be the Jacobian of system (2.1) at $z^{*}$. In this article, we assume that system (2.1) has only hyperbolic equilibria, i.e., $\operatorname{Re}(\lambda) \neq 0$, for $\lambda \in \Omega$, where $\Omega=\cup_{z^{*} \in \Gamma} \sigma\left(J\left(z^{*}\right)\right)$ and $\Gamma$ represents all equilibria of system (2.1). Also an equilibrium $z^{*}=\left(x^{*}, y^{*}\right)$ is called linear stable, if $\operatorname{Re}(\lambda)<0$ for all $\lambda \in \sigma\left(J\left(z^{*}\right)\right)$ and linearly unstable, if $\operatorname{Re}(\lambda)>0$ for at least one $\lambda \in \sigma\left(J\left(z^{*}\right)\right)$.

Lemma 2.2. Assume that system (2.2) has the following form:

$$
z_{\mathrm{k}+1}=\mathrm{G}\left(z_{\mathrm{k}}\right) \text {, }
$$

where the function $\mathrm{G}=\left[\mathrm{G}^{1}, \mathrm{G}^{2}\right]^{\top}: \mathbb{R}^{2} \longrightarrow \mathbb{R}^{2}$ is differentiable. An equilibrium point $z^{*}$ of system (2.3) is stable, if and only if all eigenvalues of $\mathrm{J}\left(z^{*}\right)$ are less than one in absolute values [11].

For describing the eigenvalues of $\mathrm{J}\left(z^{*}\right)$ we can use the following lemma.

If one wants to give some lemmas and theorems for local asymptotic stability of discrete systems, it is useful to investigate the Schur-Cohn criteria which deal with coefficient matrix of the linearized system.

Lemma 2.3. For the quadratic equation $\lambda^{2}-\operatorname{tr}\left(J\left(z^{*}\right)\right) \lambda+\operatorname{det}\left(J\left(z^{*}\right)\right)=0$, both roots satisfy $\left|\lambda_{i}\right|<1, i=1,2$, if and only if the following three conditions are satisfied:

(D1) $1+\operatorname{tr}\left(J\left(z^{*}\right)\right)+\operatorname{det}\left(J\left(z^{*}\right)\right)>0$;

(D2) $1-\operatorname{tr}\left(J\left(z^{*}\right)\right)+\operatorname{det}\left(J\left(z^{*}\right)\right)>0$;

(D3) $\operatorname{det}\left(\mathrm{J}\left(z^{*}\right)\right)<1$;

where $\operatorname{tr}\left(\mathrm{J}\left(z^{*}\right)\right)$, det $\left(\mathrm{J}\left(z^{*}\right)\right)$ denote trace and determinant of the coefficient matrix respectively. For more information one can see $[12,15]$.

Definition 2.4. The one step method (2.2) is defined nonstandard finite difference method, if at least one of the following conditions hold:

- $D_{h}\left(z_{k}\right)=\frac{z_{k+1}-z_{k}}{\varphi(h)}$, where $\varphi(h)=h+0\left(h^{2}\right)$ is a nonnegative function;

- $\mathscr{F} \mathrm{D}_{\mathrm{h}}\left(\mathrm{F} ; z_{\mathrm{k}}\right)=\mathrm{f}\left(z_{\mathrm{k}}, z_{\mathrm{k}+1}, \mathrm{~h}\right)$, where $\mathrm{f}\left(z_{\mathrm{k}}, z_{\mathrm{k}+1}, \mathrm{~h}\right)$ is a nonlocal approximation of the right hand side of system $(2.1)[2,11]$.

\section{Existence and stability of equilibria points}

We analyze the Holling Type-II Lotka Volterra predator-prey system with Allee effect which has the following type:

$$
\begin{array}{ll}
\frac{d x}{d t}=r \frac{x}{\beta+x} x(1-x)-a x y, & x(0) \geqslant 0, \\
\frac{d y}{d t}=a y(x-y), & y(0) \geqslant 0,
\end{array}
$$

where $x$ and $y$ denote the prey and predator population sizes respectively, $r, a$ and $\beta$ are constants. The 
term $\alpha(x)=\frac{x}{\beta+x}$ is called the "Allee effect" and $\beta>0$ can be called "Allee effect constant". If the term $\alpha(x)=1$ in equation (3.1), it is well-known that this equation can be called the population model without Allee effect. The larger $\beta(\beta>0)$ is, the stronger Allee effect will be [19] . It can be clearly seen that the system takes a much longer time to reach its stable steady-state solutions as $\beta$ increases. System (3.1) has the following equilibria:

(C1) $E_{1}^{*}=(0,0)$ is locally unstable;

(C2) $E_{2}^{*}=(1,0)$ is again locally unstable; and

(C3) $E_{3}^{*}=\left(\frac{r-a \beta}{a+r}, \frac{r-a \beta}{a+r}\right)$ is always stable [19].

To present a stability analysis of steady-state solutions of a discrete time Holling Type-II Lotka Volterra predator-prey system with Allee effect, the continuous nonlinear differential equation system (3.1) will be discretizated as follows:

$$
\begin{aligned}
& x^{2} \rightarrow x_{k} x_{k}, \\
& x^{3} \rightarrow x_{k} x_{k} x_{k+1} \\
& x y \rightarrow x_{k+1} y_{k}, \\
& y x \rightarrow y_{k} x_{k} \\
& y^{2} \rightarrow y_{k} y_{k+1} \\
& \frac{d x}{d t} \rightarrow \frac{x_{k+1}-x_{k}}{\varphi(h)}, \\
& \frac{d y}{d t} \rightarrow \frac{y_{k+1}-y_{k}}{\varphi(h)},
\end{aligned}
$$

where $\varphi(h)$ called denominator function depends on the step size $\Delta t=h$ and satisfies $\phi(h)=h+O\left(h^{2}\right)$. Detailed information about how to find different denominator functions can be seen in $[4,12,15,20,26]$.

Equation (3.1) is rewritten by using the discretization in equation (3.2)

$$
\begin{aligned}
& \frac{x_{k+1}-x_{k}}{\varphi(h)}=r \frac{x_{k}}{\beta+x_{k}} x_{k}\left(1-x_{k+1}\right)-a x_{k+1} y_{k}, \\
& \frac{y_{k+1}-y_{k}}{\varphi(h)}=a y_{k}\left(x_{k}-y_{k+1}\right) .
\end{aligned}
$$

Let us indicate $h_{1}=\varphi(h)=\frac{\phi(h q)}{q}$ where for some $q>Q, Q>0$ and $0<h_{1}<\frac{1}{Q}$. If $x_{k+1}$ and $y_{k+1}$ are explicitly solve from equation (3.3), following iterations can be obtained:

$$
\begin{aligned}
x_{k+1} & =\frac{x_{k}\left(\beta+x_{k}\right)+r h_{1} x_{k}^{2}}{\beta+x_{k}+\left(\beta+x_{k}\right) a h_{1} y_{k}+r h_{1} x_{k}^{2}}, \\
y_{k+1} & =\frac{\left(1+a h_{1} x_{k}\right) y_{k}}{1+a h_{1} y_{k}} .
\end{aligned}
$$

Similarly, using the discretizations of $x \rightarrow x_{k}, x^{2} \rightarrow x_{k} x_{k+1}, x y \rightarrow x_{k+1} y_{k}$ in the model without Allee effect, we can obtain $x_{k+1}$ and $y_{k+1}$ as follows:

$$
\begin{aligned}
& x_{k+1}=\frac{x_{k}\left(1+h_{2} r\right)}{1+h_{2} r x_{k}+a h_{2} y_{k}}, \\
& y_{k+1}=\frac{\left(1+a h_{2} x_{k}\right) y_{k}}{1+a h_{2} y_{k}},
\end{aligned}
$$

where the different denominator function $h_{2}$ has the same conditions of $h_{1}$. And the predator-prey systems with logistic intrinsic growth rate of the prey was studied detailed in [14]. 
Since the constants $a, r$ and $\beta$ are positive then the system (3.4) is positive and its fixed points are the equilibria of system (3.1).

We take $x_{k+1}$ is equivalent to $x_{k}$ and then

$$
\begin{aligned}
x_{k+1} & =\frac{x_{k}\left(\beta+x_{k}\right)+r h_{1} x_{k}^{2}}{\beta+x_{k}+\left(\beta+x_{k}\right) a h_{1} y_{k}+r h_{1} x_{k}^{2}}=x_{k}, \\
r x_{k} & =\left(\beta+x_{k}\right) a y_{k}+r x_{k}^{2} .
\end{aligned}
$$

Similarly we take $y_{k+1}$ is equivalent to $y_{k}$ and then

$$
\begin{gathered}
y_{k+1}=\frac{\left(1+a h_{1} x_{k}\right) y_{k}}{1+a h_{1} y_{k}}=y_{k}, \\
1+a h_{1} x_{k}=1+a h_{1} y_{k}, \\
x_{k}=y_{k} .
\end{gathered}
$$

So an equilibrium $\left(x^{*}, y^{*}\right)$ is satisfying $x^{*}=y^{*}$ and $r x^{*}=\left(\beta+x^{*}\right) a y^{*}+r x^{* 2}$.

Now we examine the stability of fixed points using Lemma 2.2. The Jacobian J of system (3.4) has the form $J(x, y)=\left(j_{j j}(x, y)\right)_{2 \times 2}$, where

$$
\begin{aligned}
J(x, y)= & \left(\begin{array}{ll}
\frac{\partial x_{k+1}}{\partial x_{k}} & \frac{\partial x_{k+1}}{\partial y_{k}} \\
\frac{\partial y_{k+1}}{\partial x_{k}} & \frac{\partial y_{k+1}}{\partial y_{k}}
\end{array}\right)=\left(\begin{array}{ll}
j_{11}(x, y) & j_{12}(x, y) \\
j_{21}(x, y) & j_{22}(x, y)
\end{array}\right), \\
j_{11}(x, y)= & \frac{\left(2 x_{k}+\beta+2 x_{k} r h_{1}\right) \cdot\left[x_{k}+\beta+\left(x_{k}+\beta\right) y_{k} a h_{1}+x_{k}^{2} r h_{1}\right]}{\left[x_{k}+\beta+\left(x_{k}+\beta\right) y_{k} a h_{1}+x_{k}^{2} r h_{1}\right]^{2}} \\
& -\frac{\left(1+y_{k} a h_{1}+2 x_{k} r h_{1}\right) \cdot\left[x_{k}\left(x_{k}+\beta\right)+x_{k}^{2} r h_{1}\right]}{\left[x_{k}+\beta+\left(x_{k}+\beta\right) y_{k} a h_{1}+x_{k}^{2} r h_{1}\right]^{2}}, \\
j_{12}(x, y)= & \frac{-\left(x_{k}+\beta\right) a h_{1}\left[x_{k}\left(x_{k}+\beta\right)+x_{k}^{2} r h_{1}\right]}{\left[x_{k}+\beta+\left(x_{k}+\beta\right) y_{k} a h_{1}+x_{k}^{2} r h_{1}\right]^{2}} \\
j_{21}(x, y)= & \frac{y_{k} a h_{1}\left(1+y_{k} a h_{1}\right)}{\left(1+y_{k} a h_{1}\right)^{2}}=\frac{y_{k} a h_{1}}{1+y_{k} a h_{1}},
\end{aligned}
$$

and

$$
\begin{aligned}
j_{22}(x, y) & =\frac{\left(1+x_{k} a h_{1}\right)\left(1+y_{k} a h_{1}\right)-a h_{1} y_{k}\left(1+x_{k} a h_{1}\right)}{\left(1+y_{k} a h_{1}\right)^{2}}, \\
& =\frac{1+x_{k} a h_{1}}{\left(1+y_{k} a h_{1}\right)^{2}} .
\end{aligned}
$$

Theorem 3.1. The fixed point $\mathrm{E}_{1}^{*}=(0,0)$ is a nonhyperbolic fixed point.

Proof. Eigenvalues of $\mathrm{J}$ at the equilibrium $(0,0)$ are located as follows:

$$
\begin{gathered}
J(0,0)=\left(\begin{array}{ll}
1 & 0 \\
0 & 1
\end{array}\right) \\
|\lambda I-J|=\left|\begin{array}{cc}
\lambda-1 & 0 \\
0 & \lambda-1
\end{array}\right|=(\lambda-1)(\lambda-1)=0
\end{gathered}
$$


So eigenvalues of $\mathrm{J}$ at the equilibrium $(0,0)$ are $\lambda_{1}=1$ and $\lambda_{2}=1$ and $\left|\lambda_{1}\right|=1,\left|\lambda_{2}\right|=1$, i.e., $E_{1}^{*}=(0,0)$ is always nonhyperbolic fixed point which is consistent with the dynamical behaviour of equation (3.1).

Using that $r x_{k}=\left(\beta+x_{k}\right) a y_{k}+r x_{k}^{2}$ and the fact that $y_{k}=0$, we find $x_{k}$ as follows:

$$
\begin{gathered}
r x_{k}=\left(\beta+x_{k}\right) a y_{k}+r x_{k}^{2}, \\
x_{k}=1 .
\end{gathered}
$$

Theorem 3.2. The fixed point $\mathrm{E}_{2}^{*}=(1,0)$ is always unstable and a saddle point.

Proof. Eigenvalues of $\mathrm{J}$ at the equilibrium $\mathrm{E}_{2}^{*}=(1,0)$ are located as follows:

$$
\begin{gathered}
J(1,0)=\left(\begin{array}{cc}
\frac{1+\beta}{\left(1+\beta+r h_{1}\right)} & \frac{-(1+\beta) a h_{1}}{\left(1+\beta+r h_{1}\right)} \\
0 & 1+a h_{1}
\end{array}\right), \\
|\lambda I-J|=\left|\begin{array}{cc}
\lambda-\frac{1+\beta}{\left(1+\beta+r h_{1}\right)} & \frac{(1+\beta) a h_{1}}{\left(1+\beta+r h_{1}\right)} \\
0 & \lambda-\left(1+a h_{1}\right)
\end{array}\right|=\left(\lambda-\frac{1+\beta}{\left(1+\beta+r h_{1}\right)}\right)\left(\lambda-1-a h_{1}\right)=0 .
\end{gathered}
$$

So eigenvalues of $J$ at the equilibrium $(1,0)$ are $\lambda_{1}=\frac{1+\beta}{\left(1+\beta+r h_{1}\right)}$ and $\lambda_{2}=1+a h_{1},\left|\lambda_{1}\right|<1$ and $\left|\lambda_{2}\right|>1$, for all $h_{1}>0$, the fixed point $E_{2}^{*}$ is always unstable and a saddle point.

Theorem 3.3. Assume that the fixed point $\mathrm{E}_{3}^{*}$ is a hyperbolic fixed point i,e., $\operatorname{Re}(\lambda) \neq 0$ for all $\lambda$. The fixed point $\mathrm{E}_{3}^{*}$ is locally asymptotically stable, if and only if three conditions of Lemma 2.3 satisfy and $\left(\mathrm{x}^{*}, \mathrm{y}^{*}\right)$ is unstable, if at least one of the conditions is not satisfied.

Proof. Using the equations $x^{*}=y^{*}$ and $r x^{*}=\left(\beta+x^{*}\right) a y^{*}+r x^{* 2}$, the Jacobian $J\left(x^{*}, y^{*}\right)=\left(j_{j j}\left(x^{*}, y^{*}\right)\right)_{2 \times 2}$ at the fixed point $\left(x^{*}, y^{*}\right)$ can be found as follows:

$$
\begin{aligned}
& j_{11}\left(x^{*}, y^{*}\right)=\frac{x^{*}+\beta+2 \operatorname{rh}_{1} x^{*}-a h_{1} x^{* 2}-2 \operatorname{rh}_{1} x^{* 2}}{x^{*}+\beta+r h_{1} x^{*}}, \\
& j_{12}\left(x^{*}, y^{*}\right)=\frac{-a h_{1} x^{*}\left(x^{*}+\beta\right)}{x^{*}+\beta+\operatorname{rh}_{1} x^{*}}, \\
& j_{21}\left(x^{*}, y^{*}\right)=\frac{y^{*} a h_{1}}{1+y^{*} a h_{1}}=\frac{x^{*} a h_{1}}{1+x^{*} a h_{1}},
\end{aligned}
$$

and

$$
\begin{aligned}
j_{22}\left(x^{*}, y^{*}\right) & =\frac{1}{1+x^{*} a h_{1}}, \\
J^{*} & =\left(\begin{array}{cc}
\frac{x^{*}+\beta+2 r h_{1} x^{*}-a h_{1} x^{* 2}-2 r h_{1} x^{* 2}}{x^{*}+\beta+r h_{1} x^{*}} & \frac{-a h_{1} x^{*}\left(x^{*}+\beta\right)}{x^{*}+\beta+r h_{1} x^{*}} \\
\frac{a h_{1} x^{*}}{1+a h_{1} x^{*}} & \frac{1}{1+a h_{1} x^{*}}
\end{array}\right) .
\end{aligned}
$$

Eigenvalues $\lambda_{1}$ and $\lambda_{2}$ of $J\left(x^{*}, y^{*}\right)$ are roots of the quadratic equation in Lemma 2.3:

$$
\lambda^{2}-\operatorname{tr}\left(J\left(z^{*}\right)\right) \lambda+\operatorname{det}\left(J\left(z^{*}\right)\right)=0,
$$

where

$$
\operatorname{tr}\left(J\left(z^{*}\right)\right)=\frac{2 x^{*}+2 \beta+3 r h_{1} x^{*}-2 r h_{1} x^{* 2}+a \beta h_{1} x^{*}+2 a r h_{1}^{2} \chi^{* 2}-a^{2} h_{1}^{2} \chi^{* 3}-2 a r h_{1}^{2} x^{* 3}}{\left(x^{*}+\beta+r h_{1} x^{*}\right) \cdot\left(1+a h_{1} x^{*}\right)}
$$


and

$$
\operatorname{det}\left(J\left(z^{*}\right)\right)=\frac{x^{*}+\beta+2 r h_{1} x^{*}-a h_{1} \chi^{* 2}-2 r h_{1} x^{* 2}+a^{2} \beta h_{1}^{2} \chi^{* 2}+a^{2} h_{1}^{2} \chi^{* 3}}{\left(x^{*}+\beta+r h_{1} x^{*}\right) \cdot\left(1+a h_{1} x^{*}\right)} .
$$

Note that $\operatorname{tr}\left(J\left(z^{*}\right)\right)=\operatorname{tr}\left(J\left(z^{*}\right)\right)\left(h_{1}\right)$ and $\operatorname{det}\left(J\left(z^{*}\right)\right)=\operatorname{det}\left(J\left(z^{*}\right)\right)\left(h_{1}\right)$ are continuous functions of $h_{1}\left(h_{1}>0\right)$. Also, $\operatorname{tr}\left(J\left(z^{*}\right)\right)(0)=2$ and $\operatorname{det}\left(J\left(z^{*}\right)\right)(0)=1$, which implies that there exist constants $\widetilde{\mathrm{K}}_{\left(x^{*}, y^{*}\right)}>0$ such that $1+\operatorname{tr}\left(\mathrm{J}\left(z^{*}\right)\right)\left(\mathrm{h}_{1}\right)+\operatorname{det}\left(\mathrm{J}\left(z^{*}\right)\right)\left(\mathrm{h}_{1}\right)>0$, for all $0<\mathrm{h}_{1}<\widetilde{\mathrm{K}}_{\left(x^{*}, y^{*}\right)}$.

If we want to determine a specific value for Q (in Remark 3.4) we need to evaluate the constants $\widetilde{\mathrm{K}}_{\left(x^{*}, y^{*}\right)}$. After some calculations as the following:

$$
1+\operatorname{tr}\left(J\left(z^{*}\right)\right)\left(h_{1}\right)+\operatorname{det}\left(J\left(z^{*}\right)\right)\left(h_{1}\right)>0 \Leftrightarrow B h_{1}^{2}+C h_{1}+4 \beta+4 x^{*}>0,
$$

where

$$
\mathrm{B}=3 a r x^{* 2}+a^{2} \beta x^{* 2}-2 a r x^{* 3} \text { and } C=6 r x^{*}+2 a \beta x^{*}-4 r x^{* 2} .
$$

Therefore, the constant $\widetilde{\mathrm{K}}_{\left(x^{*}, y^{*}\right)}$ can be selected as follows:

$$
\widetilde{\mathrm{K}}_{\left(x^{*}, y^{*}\right)}=\left\{\begin{array}{cl}
\frac{2 \sqrt{|\beta|}+2 \sqrt{\left|x^{*}\right|}}{\sqrt{|\beta|}}, & C=0, \\
\frac{4 \beta+4 x^{*}}{|C|}, & B=0, \\
\min \left(\frac{|C|}{|B|}, \frac{2 \sqrt{|\beta|}+2 \sqrt{\left|x^{*}\right|}}{|C|}\right), & \text { otherwise. }
\end{array}\right.
$$

Let us suppose that $\left(x^{*}, y^{*}\right)$ is a stable fixed point of equation (3.1). Then,

$$
1-\operatorname{tr}\left(J\left(z^{*}\right)\right)+\operatorname{det}\left(J\left(z^{*}\right)\right)=\frac{h_{1}^{2}}{\left(\beta+x^{*}+r h_{1} x^{*}\right) \cdot\left(1+a h_{1} x^{*}\right)}\left(2 a r x^{* 3}-a r x^{* 2}+2 a^{2} x^{* 3}+a^{2} \beta x^{* 2}\right)>0,
$$

and condition (D2) of Lemma 2.3 holds. The last condition (D3) of Lemma 2.3 is equivalent to

$$
\begin{gathered}
\operatorname{det}\left(J\left(z^{*}\right)\right)-1=\frac{r h_{1} x^{*}-2 a h_{1} x^{* 2}-2 r h_{1} x^{* 2}+a^{2} \beta h_{1}^{2} \chi^{* 2}+a^{2} h_{1}^{2} \chi^{* 3}-a \beta h_{1} x^{*}-a r h_{1}^{2} \chi^{* 2}}{\left(\beta+x^{*}+r h_{1} x^{*}\right) \cdot\left(1+a h_{1} x^{*}\right)}<0, \\
\frac{h_{1}^{2}\left(a^{2} \beta x^{* 2}+a^{2} \chi^{* 3}-a r x^{* 2}\right)}{\left(\beta+\chi^{*}+r h_{1} \chi^{*}\right) \cdot\left(1+a h_{1} \chi^{*}\right)}+h_{1} \cdot \frac{\left(r x^{*}-2 a x^{* 2}-2 r \chi^{* 2}-a \beta x\right)}{\left(\beta+x^{*}+r h_{1} x^{*}\right) \cdot\left(1+a h_{1} \chi^{*}\right)}<0 .
\end{gathered}
$$

The inequality (3.6) is true when $h_{1}<K_{\left(x^{*}, y^{*}\right)}$, where

$$
K_{\left(x^{*}, y^{*}\right)}=\frac{\left|a^{2} \beta x^{* 2}+a^{2} \chi^{* 3}-a r x^{* 2}\right|}{\left|r x^{*}-2 a x^{* 2}-2 r x^{* 2}-a \beta x\right|} .
$$

Therefore, if $h_{1}<\min \left(K_{\left(x^{*}, y^{*}\right)}, \widetilde{K}_{\left(x^{*}, y^{*}\right)}\right)$, conditions (D1), (D2) and (D3) of Lemma 2.3 hold and $\left(x^{*}, y^{*}\right)$ is a stable fixed point of system (3.3).

Remark 3.4. If we select

$$
\mathrm{Q}=\max _{\left(x^{*}, y^{*}\right) \in \widetilde{\Gamma}}\left(\frac{1}{\mathrm{~K}_{\left(x^{*}, y^{*}\right)}}, \frac{1}{\widetilde{\mathrm{K}}_{\left(x^{*}, y^{*}\right)}}\right),
$$

where $\widetilde{\Gamma}$ represents the set of all equilibria of equation (3.1), then

$$
\mathrm{h}_{1}<\min \left(\mathrm{K}_{\left(x^{*}, y^{*}\right)}, \widetilde{\mathrm{K}}_{\left(x^{*}, \mathrm{y}^{*}\right)}\right),
$$

for every equilibrium $\left(x^{*}, y^{*}\right)$, which guarantee dynamical consistence between the differential equation system (3.1) and the numerical scheme (3.3) around all equilibria. 
Corollary 3.5. Let $\phi$ be a real-valued function on $R$ that satisfies the property:

$$
\phi(h)=h+0\left(h^{2}\right), \quad \text { and } 0<\phi(h)<1, \quad \forall h>0 .
$$

There exists a constant $\mathrm{Q}>0$ such that $\varphi(\mathrm{h})$ has the form $\varphi(\mathrm{h})=\frac{\phi(\mathrm{hq})}{\mathrm{q}}$ for some $\mathrm{q}>\mathrm{Q}$ and $0<\mathrm{h}_{1}<\frac{1}{\mathrm{Q}}$.

\section{Numerical simulations}

In this section parameters are set as $h=0.01, \beta=0.05, r=2.5$ and $a=17$. The denominator functions $h_{1}, h_{2}$ can be selected as $\frac{e^{a h}-1}{a}, \frac{e^{r h}-1}{r}$ respectively. The initial conditions are taken as $x(0)=$ 0.005 and $y(0)=0.01$, which are initial prey and predator densities, respectively [19]. We give some numerical simulations of the Holling Type II Lotka-Volterra predator-prey model with Allee effect defined by equation (3.3). We used the MAPLE for these computations. The steady-state solutions of the systems corresponding to these parameter values are $x^{*}=y^{*} \cong 0.0846$.

Eigenvalues of $J$ at the equilibrium $(0,0)$ are $\lambda_{1}=1$ and $\lambda_{2}=1$, i.e. the equilibrium point $(0,0)$ is nonhyperbolic fixed point. When the eigenvalues of the Jacaobian matrix of the linearized system at $(0,0)$ are equal 1 , the stability is undetermined immediately and needs to investigate the center manifold ot the Niemark-Sacker bifurcation. But in this paper we are interested in only nonhyperbolic fixed points.

Eigenvalues of $J$ at the equilibrium $(1,0)$ are located, are $\lambda_{1} \cong 0.974703$ and $\lambda_{2} \cong 1.185304$. The equilibrium point $(1,0)$ is locally unstable.

Let us now analyze the stability of the positive steady-state solution $\left(x^{*}, y^{*}\right)$. The Jacobian matrix $J\left(x^{*}, y^{*}\right)$ can be simplified as follows:

$$
\begin{gathered}
\operatorname{tr}(M)=1.990577, \\
\operatorname{det}(M)=0.990722<1, \\
1-\operatorname{tr}(M)+\operatorname{det}(M)=0.000145>0, \\
1+\operatorname{tr}(M)+\operatorname{det}(M)=3.981300>0 .
\end{gathered}
$$

The three conditions of Lemma 2.3 are satisfied equilibrium point $\left(x^{*}, y^{*}\right)$ is locally unstable. $K_{\left(x^{*}, y^{*}\right)} \cong$ $0.184679, \mathrm{~h}_{1} \cong 0.0109002$.

Similar calculations will be done for other values of $\beta$.

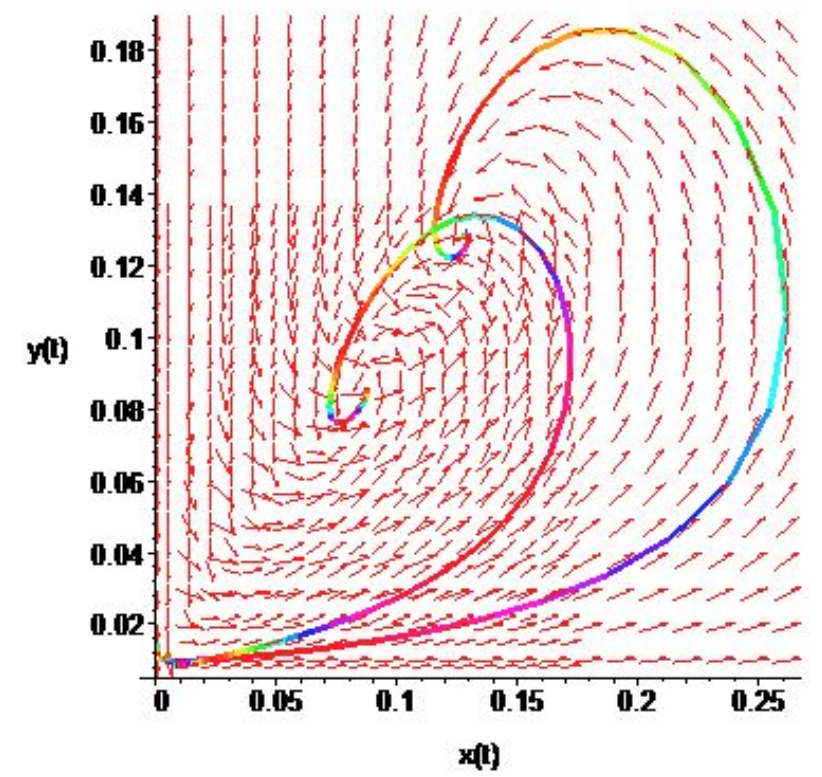

Figure 1: Phase portrait of equation (3.1) with and without Allee effects. 


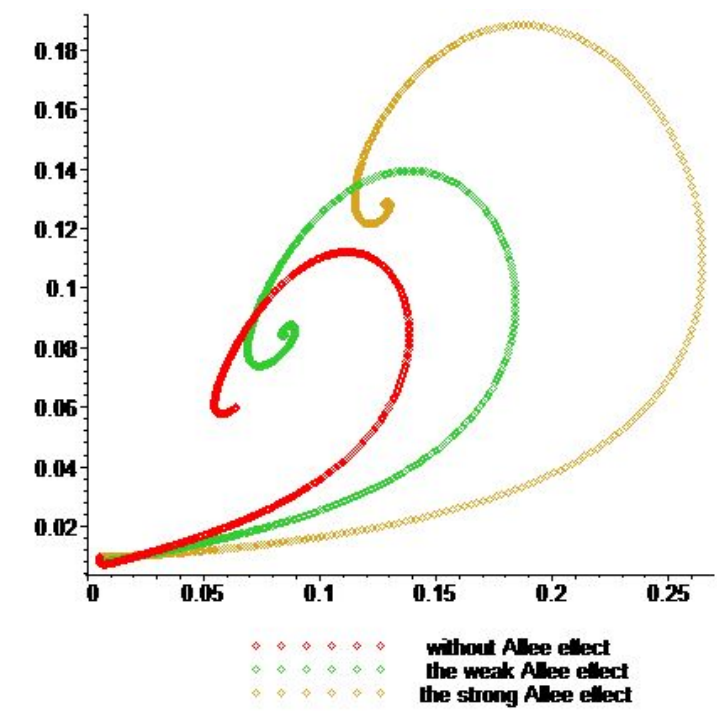

Figure 2: Numerical approximations of the solutions of equations (3.4) and (3.5) with $\beta=0.05$ for the weak Allee effect and $\beta=0.07$ for the strong Allee effect and without Allee effect.

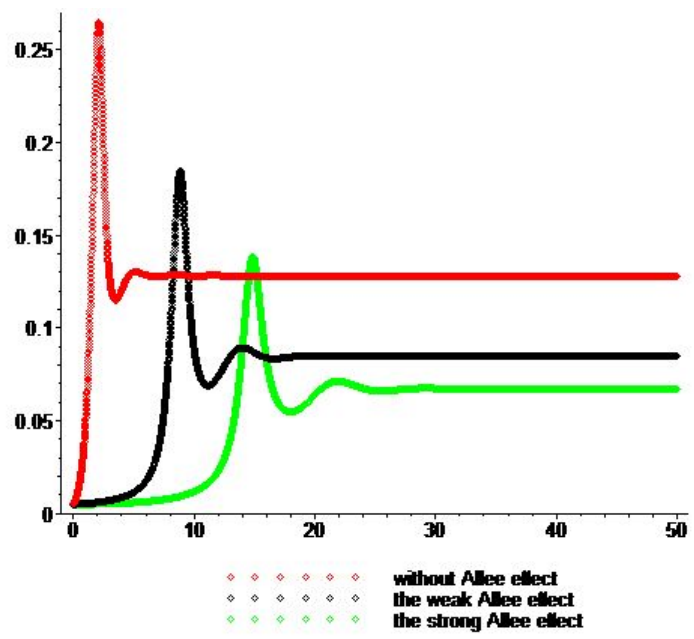

Figure 3: Numerical approximations of the prey densities with $\beta=0.05, \beta=0.07$ and without Allee effect.

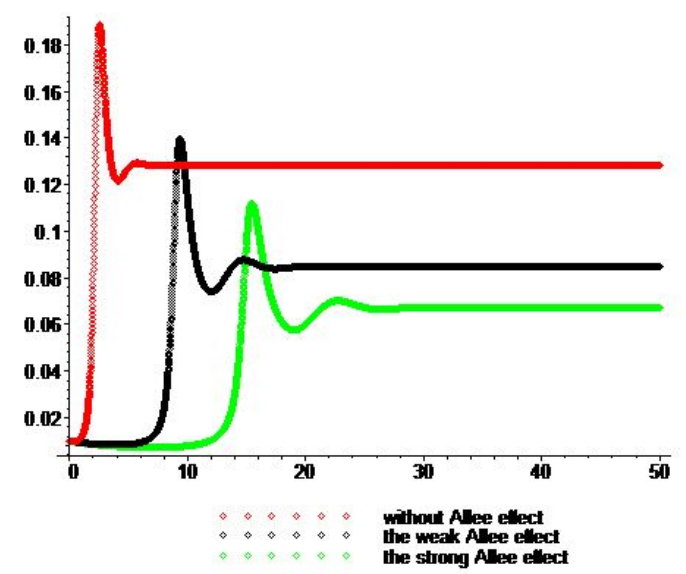

Figure 4: Numerical approximations of the predator densities with $\beta=0.05, \beta=0.07$ and without Allee effect. 


\section{Conclusions}

In this study, NSFD were developed for a general class of Holling Type-II Lotka Volterra predator-prey system with and without Allee effect. The new NSFD method preserves the positivity of solutions with positive initial conditions and the physical and biological properties, especially the stability of equilibria of the approximated predator-prey system. This system with general functional response has an Allee effect on prey population. Stabilizer power of Allee effect has mentioned and the Allee effect on prey population for a discretizated predator-prey systems has studied. Strong and weak Allee effect have mentioned that as a result of the Allee effect has a huge impact for deterioration of biological balance.

This article has focused on the stability analysis of a discretized predator-prey system subject to an Allee effect on the prey population. We first supplied some definitions and preliminary results. The larger $\beta,(\beta>0)$ is the stronger the Allee effect will be. In addition, numerical simulations show that the system takes a much longer time to reach its stable steady-state solutions as $\beta$ increases in the Allee effect. We also used the Schur-Cohn criteria which deal with coefficients of the characteristic polynomial for determining the stability of discrete-time system.

\section{References}

[1] H. N. Agiza, E. M. ELabbasy, H. EL-Metwally, A. A. Elsadany, Chaotic dynamics of a discrete prey-predator model with Holling type II, Nonlinear Anal. Real World Appl., 10 (2009), 116-129. 1

[2] R. Anguelov, J. M. S. Lubuma, Contributions to the mathematics of the nonstandard finite difference method and applications, Numer. Methods Partial Differential Equations, 17 (2001), 518-543. 1, 2.4

[3] A. Atangana, I. Koca, Chaos in a simple nonlinear system with Atangana-Baleanu derivatives with fractional order, Chaos Solitons Fractals, 89 (2016), 447-454. 1

[4] N. Bairagi, M. Biswas, A predator-prey model with Beddington-DeAngelis functional response: a non-standard finitedifference method, J. Difference Equ. Appl., 4 (2016), 529-541. 1, 3

[5] F. Berezovskaya, G. Karev, R. Arditi, Parametric analysis of the ratio-dependent predator-prey model, J. Math. Biol., 43 (2001), 221-246. 1

[6] Q.-Y. Bie, Q.-R. Wang, Z.-A. Yao, Cross-diffusion induced instability and pattern formation for a Holling type-II predatorprey model, Appl. Math. Comput., 247 (2014), 1-12. 1

[7] C. Çelik, O. Duman, Allee effect in a discrete-time predator-prey system, Chaos Solitons Fractals, 40 (2009), $1956-1962$. 1

[8] D. T. Dimitrov, H. V. Kojouharov, Nonstandard finite-difference schemes for general two-dimensional autonomous dynamical systems, Appl. Math. Lett., 18 (2005), 769-774. 1

[9] D. T. Dimitrov, H. V. Kojouharov, Positive and elementary stable nonstandard numerical methods with applications to predator-prey models, J. Comput. Appl. Math., 189 (2006), 98-108. 1, 2

[10] D. T. Dimitrov, H. V. Kojouharov, Nonstandard numerical methods for a class of predator-prey models with predator interference, Proceedings of the Sixth Mississippi State-UBA Conference on Differential Equations and Computational Simulations, Electron. J. Differ. Equ. Conf., Southwest Texas State Univ., San Marcos, TX, 15 (2007), 67-75. 1

[11] D. T. Dimitrov, H. V. Kojouharov, Nonstandard finite-difference methods for predator-prey models with general functional response, Math. Comput. Simulation, 78 (2008), 1-11. 1, 2.2, 2.4

[12] S. N. Elaydi, An introduction to difference equations, Second edition, Undergraduate Texts in Mathematics, SpringerVerlag, New York, (1999). 2.3, 3

[13] A. Gkana, L. Zachilas, Incorporating prey refuge in a prey-predator model with a Holling type I functional response: random dynamics and population outbreaks, J. Biol. Phys., 39 (2013), 587-606. 1

[14] C. S. Holling, The functional response of predators to prey density and its role in mimicry and population regulation, Mem. Entomol. Soc. Can., 97 (1965), 5-60. 1, 3

[15] S. Jang, S. Elaydi, Difference equations from discretization of a continuous epidemic model with immigration of infectives, Can. Appl. Math. Q., 11 (2004), 93-105. 2.3, 3

[16] H. Jansen, E. H. Twizell, An unconditionally convergent discretization of the SEIR model, Math. Comput. Simulation, 58 (2002), 147-158. 1

[17] Y. Kuang, E. Beretta, Global qualitative analysis of a ratio-dependent predator-prey system, J. Math. Biol., 36 (1998), 389-406. 1

[18] J. M. S. Lubuma, A. Roux, An improved theta-method for systems of ordinary differential equations, Dedicated to Professor Ronald E. Mickens on the occasion of his 60th birthday, J. Difference Equ. Appl., 9 (2003), 1023-1035. 1

[19] H. Merdan, Stability analysis of a Lotka-Volterra type predator-prey system involving Allee effects, ANZIAM J., 52 (2010), 139-145. 1, 3, 4

[20] R. E. Mickens, Nonstandard finite difference models of differential equations, World Scientific Publishing Co., Inc., River Edge, NJ, (1994). 1, 3 
[21] J. D. Murray, Mathematical biology, Second edition, Biomathematics, Springer-Verlag, Berlin, (1993). 1

[22] M. Y. Ongun, D. Arslan, R. Garrappa, Nonstandard finite difference schemes for a fractional-order Brusselator system, Adv. Difference Equ., 2013 (2013), 13 pages. 1

[23] M. Y. Ongun, I. Turhan, A numerical comparison for a discrete HIV infection of $\mathrm{CD}^{+} \mathrm{T}-\mathrm{Cell}$ model derived from nonstandard numerical scheme, J. Appl. Math., 2013 (2013), 9 pages.

[24] W. Piyawong, E. H. Twizell, A. B. Gumel, An unconditionally convergent finite-difference scheme for the SIR model, Appl. Math. Comput., 146 (2003), 611-625. 1

[25] M. Sen, M. Banarjee, A. Morozov, Bifurcation analysis of a ratio-dependent preypredator model with the Allee effect, Ecol. Complex., 11 (2013), 12-27. 1

[26] S. Sharma, G. P. Samanta, A ratio-dependent predator-prey model with Allee effect and disease in prey, J. Appl. Math. Comput., 47 (2005), 345-364. 3

[27] R.-Z. Yang, J.-J. Wei, Stability and bifurcation analysis of a diffusive prey-predator system in Holling type III with a prey refuge, Nonlinear Dynam., 79 (2015), 631-646. 1

[28] S.-R. Zhou, Y.-F. Liu, G. Wang, The stability of predatorprey systems subject to the Allee effects, Theor. Popul. Biol., 67 (2005), 23-31. 1 